\title{
THE EFFECT OF A CONCUSSION ON THE HAZARD ANTICIPATION ABILITY IN TEEN DRIVERS
}

\author{
Atefeh Katrahmani, Matthew Romoser \\ Western New England University \\ Springfield, MA, USA \\ Email: atefeh.katrahmani@gmail.com
}

\begin{abstract}
Summary: Driving after a brain injury is controversial. Since diagnosing a concussion and tracking the healing trend is challenging, whether or not a patient is fit-to-drive after the injury is open to interpretation. The primary purpose of the present research was to investigate the effect of a concussion on teen drivers' hazard anticipation skill. Twenty-four participants were recruited for this study in two groups: the concussed teen driver group and the non-concussed teen driver group. They were asked to wear an eye-tracker and drive with a driving simulator. The drive included several scenarios with potential latent hazards. While driving, the participants were expected to scan the latent hazards with their eyes and fixate at the hazardous area. The results show significant differences $(\mathrm{p}<0.05)$ in the hazard anticipation skills between the two groups on two primary aspects: 1). The concussed group showed more random eye movements while the non-concussed participants had more deliberate eye fixations with less distractions and saccadic jumping. 2) The concussed patients showed a significantly poorer performance in anticipating the potential hazards. In conclusion, results indicate concussions can affect the hazard prediction skills of the teens, which in turn makes the driving task riskier for this group of drivers.
\end{abstract}

\section{INTRODUCTION}

Based on statistics from United States' emergency rooms, it is estimated that annually there are between 1.4 to 3.8 million sports-related injuries that result in mTBI (mild Traumatic Brain Injury) (Bazarian, Veazie, Mookerjee, \& Lerner, 2006; Halstead \& Walter, 2010; Langlois, Rutland-Brown, \& Thomas, 2006; Torres et al., 2013). Concussion, a subset of Mild Traumatic Brain Injury (mTBI), occurs from a sudden biomechanical force on the brain, which is injured by the sudden impact against the skull. Diagnosis and assessment of healing of concussions is complicated, because assessment tools are subjective, and results are open to interpretation.

A further complication is caused by the resistance of many patients to receiving the diagnosis of a concussion, once they understand that the diagnosis may limit their ability to return to activities such as driving and playing sports (Chrisman, Quitiquit, \& Rivara, 2013). A study of professional athletes who suffer a concussion revealed that almost $43 \%$ of the members of this population concealed their symptoms to prevent limitations on athletic participation (RegisterMihalik et al., 2013; Torres et al., 2013).

Driving is a complex task which needs physical and cognitive skills functioning together. It needs the ability to receive sensory information, process the information, and to make proper, timely judgments and responses (Gregory, 1981). Any malfunction of physical or cognitive skills may reduce driving performance (Wiebo H. Brouwer \& Withaar, 1997; Shenouda, Hendrickson, 
Davenport, Barber, \& Bell, 2012; Webster, Rapport, Godlewski, \& Abadee, 1994), slow reaction time (Stokx \& Gaillard, 1986), reduce driving skill (Stokx \& Gaillard, 1986), and increase crash risk. Research shows that many cognitive processes, which are necessary for driving, will be affected by concussion (Bottari, Lamothe, Gosselin, Gélinas, \& Ptito, 2012; Preece, Horswill, \& Geffen, 2010). Not only would concussion affect the driving performance, but it also may exacerbate concussion symptoms (Rose, Weber, Collen, \& Heyer, 2015).

Since the diagnosis of concussion and healing duration can be controversial, there has not been a particular method used to assess whether the patient is fit-to-drive. Many clinicians, patients and parents do not know that driving after suffering a concussion would be potentially dangerous (Preece, Geffen, \& Horswill, 2013). Decision on driving after injury depends on the primary care physician's interpretation of the patient's progress. They might take a risk and let the patient drive or might be conservative and recommend the patient not drive even when the patient is partially safe to drive. In the case of fit-to-drive assessment, no research has been done. Most existing research tests the driving ability and driving performance after a concussion (Bottari et al., 2012; Novack et al., 2006; Preece et al., 2010; Sivak et al., 1981; Stokx \& Gaillard, 1986), or discusses possible residual impairments after a concussion recovery (Schneider \& Gouvier, 2005). None directly assess if a patient is fit-to-drive. The current study used a head-mounted eye-tracker to test the hazard anticipation skills of teen drivers after a concussion. The goal was to see if in comparison with the healthy teens the concussed teen patients behave and scan differently when encountering situations with potential hazards. The results of this study will further or understanding of the effect of concussions on hazard anticipation skills of the concussed teens.

\section{METHODS}

The present research seeks to provide a method to assess concussion impact on driving behavior in patients with concussion symptoms. Driving simulation scenarios were designed to assess whether or not a brain-injured participant is able to identify the hazards and react properly.

\section{Participants}

A total of 24 participants (16- 18 years old) were recruited for this study, the concussed driver cohort (12 concussed) and the non-concussed driver cohort (12 non-concussed). All participants held a driver's license at the time of the study. None of the participants were involved in the driving task while receiving a concussion. Participants with a history of other neurological or eye disorders like Parkinson's or colorblindness were excluded from the study. The concussed driver cohort participants were among the patients referred to Connecticut Children's Medical Center (CCMC) in Farmington, CT. The participants were selected based on the doctors' judges based on the severity of their injury. Their concussion symptoms were mild. Drivers in the nonconcussed cohort were selected from healthy volunteers from the Farmington area.

\section{Apparatus}

Driving Simulator. Scenarios were developed on the STISIM V3 Mobile Driver Assessment \& Training Simulator (MODATS). MODATS is a portable 3-screen simulator developed for field research in places such as driving schools, research centers and medical facilities (Figure 1). The 
simulator consists of a gaming chair and high-end plate steel and cast-iron steering and pedal controls. Three 24" LCD monitors are mounted in front of the driver providing an approximately 150 degree field of view.

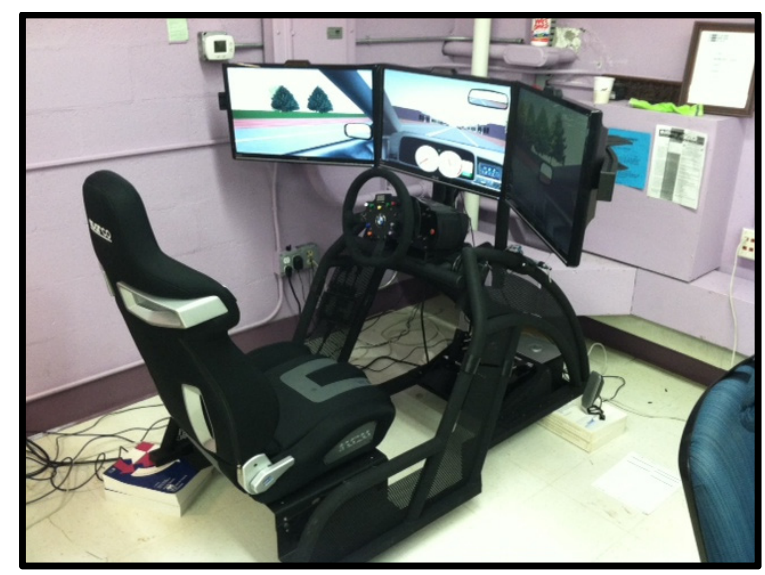

Figure 1. Mobile Driver Assessment \& Training Simulator (MODATS)

Eye-Tracker. Drivers' scanning was recorded using a Tobii Pro-II mobile eye-tracker. The eyetracker consists of a lightweight pair of glasses with the scene camera and eye cameras integrated into the frame. The system was calibrated via a wireless connection to the analysis laptop and videos were recorded on a 3 " 5 " $\times 0.5$ " video recording unit clipped to the participant's belt. The driver's point of gaze, represented as a red circle, was superimposed upon the scene camera video image.

\section{Experimental Procedure}

The study took place in a single session, lasting approximately half an hour for each participant. During this session, participants met the study administrator in a lab at CCMC. Informed consent was received at this time and the participants were given the opportunity to ask any questions. The participants were also asked to fill out a pre-study questionnaire asking about their demographic data and driving history.

After consents and questionnaires were provided, participants received instructions about what they were going to do and examples of what was expected during their driving. Participants sat in the driving simulator fitted and calibrated for the Tobii2 Mobile eye-tracker. The participant then completed a very short (30-second) practice drive on the simulator in order to become familiar with the controls and driving the vehicle through the simulated environment. This allowed the study administrator to screen for simulator sickness before the data collection began.

The study administrator asked participants to put on the eye-tracker and sit at the simulator. The study administrator ran a four-minute drive in which participants were expected to anticipate hazards, scan, and fixate at the areas with potential hazards (Figure 2). However, the hazards did not directly materialize during driving. The participants' points-of-gaze were recorded continuously throughout the drive using the eye-tracker. After the experimental drive, the study 
administrator helped the participant out of the simulator, removed the eye-tracker and the simulated drive was finished.

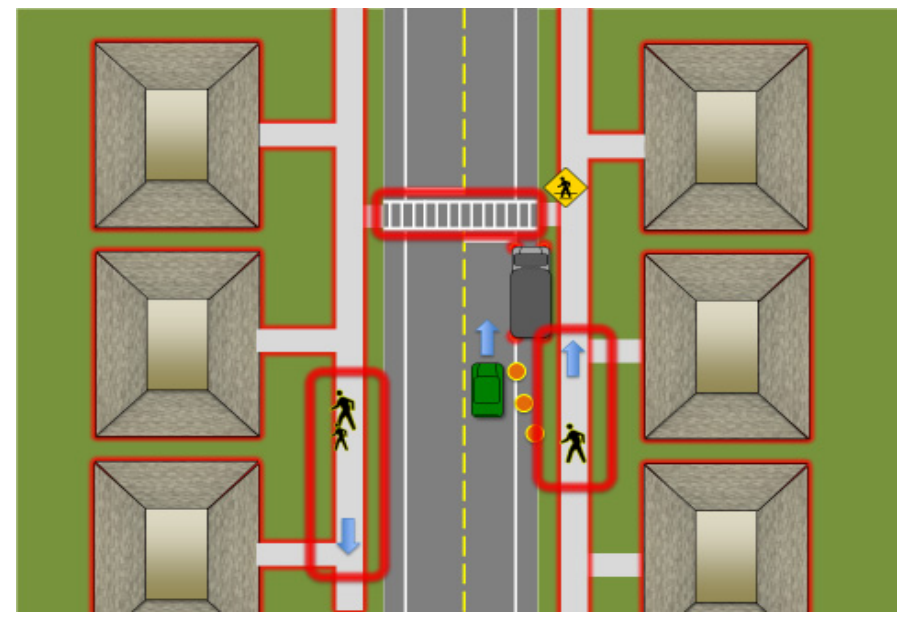

Figure 2. A top-down view of an exemplary scenario

\section{RESULTS}

In the driving simulator test, the participants were asked to wear the eye-tracker and drive several scenarios in a driving simulator. The drive consisted of a long driving scenario with six potential latent hazards. The participants were asked to drive, scan for the latent hazard and mitigate the hazards. The eye-tracking videos were used for the analysis. The participants' fixation points were recorded with the eye-tracker. The participants' fixation points were mapped and used as the analysis metrics. For instance, if the participants fixated at the hazardous area and slowed down and/or mitigate the hazard, it was assumed the participant anticipated the hazard and coded as "one". Otherwise, it was coded as "zero". Blind scoring was utilized for each participant. For each of the six scenarios, each participant received a score (zero or one) based on their hazard anticipation behavior. So, by the end of the analysis, 144 binary numbers were extracted (twentyfour participants multiplied by six scenarios).

SPSS chi-squared test results showed a significant difference between the hazard anticipation skills of the participants with concussion symptoms and the participants without concussion symptoms $\left(\chi^{2}=6.28, p=0.012\right)$. The results showed that the concussed patients were less able to recognize and fixate at the hazardous area. On the other hand, the participants without concussions symptoms were more successful in hazard anticipation and they were more able to mitigate the hazard. They also had more fixations and they fixated at the hazardous area more deliberately. 
Table 1. Chi-Square test results in SPSS for high fidelity test

\begin{tabular}{|c|c|c|c|c|c|}
\hline & Value & $\mathrm{df}$ & $\begin{array}{c}\text { Asymptotic } \\
\text { Significance } \\
\text { (2-sided) }\end{array}$ & $\begin{array}{l}\text { Exact Sig. } \\
\text { (2-sided) }\end{array}$ & $\begin{array}{c}\text { Exact Sig } \\
\text { (1-sided) }\end{array}$ \\
\hline Pearson Chi-Square & $6.280^{\mathrm{a}}$ & 1 & .012 & & \\
\hline Continuity Correction ${ }^{\mathrm{b}}$ & 5.471 & 1 & .019 & & \\
\hline Likelihood Ratio & 6.328 & 1 & .012 & & \\
\hline Fisher's Exact Test & & & & .019 & .010 \\
\hline $\mathrm{N}$ of Valid Cases & 144 & & & & \\
\hline
\end{tabular}

Figure 3 shows that the concussed participants (Group 1) showed a wider range in their ability to anticipate and mitigate hazards, whereas the non-concussed participants (Group 2) were more able to do so. On average, the concussed participants were able to anticipate almost 2.5 scenarios out of 6 latent hazards scenarios, while the non-concussed participants were able to anticipate 4 scenarios. In addition, while the non-concussed participants were able to anticipate at least half of the scenarios properly, the minimum hazard recognition number for the concussed participants were 1 scenario out of 6 .

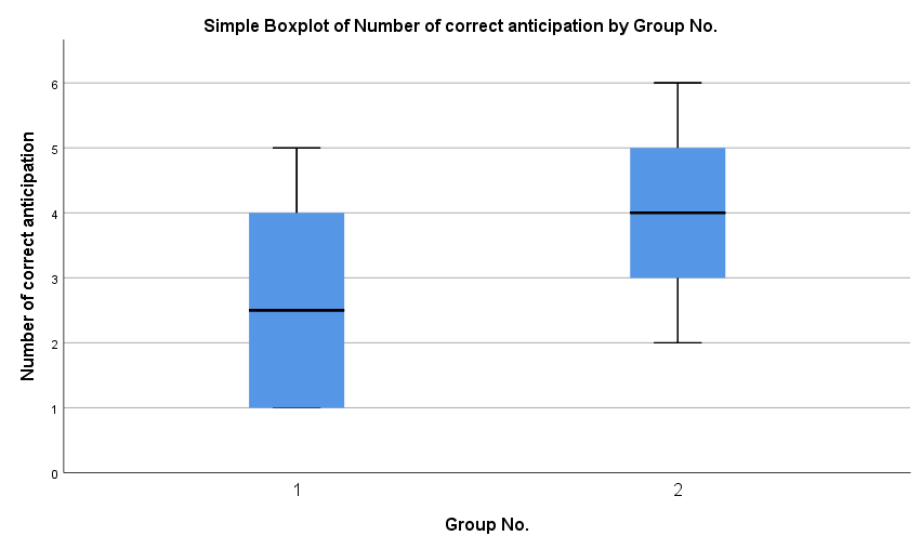

Figure 3. Number of hazard recognition

\section{SUMMARY \& CONCLUSIONS}

In general, the results demonstrate that when encountering a potential hazard, a healthy teen driver was more likely to be able to control the situation. They were more able to scan for the hazard showing greater numbers of fixations. The primary goal of this study was to investigate the effect of a concussion on hazard anticipation skills of the teen drivers. The results showed a 
significant difference between the hazard anticipation ability of the concussed and the nonconcussed participants. While the concussed participants showed difficulty recognizing the hazards, the non-concussed teens were more likely to scan and focus at the hazardous area.

Eye tracking records demonstrated a significant difference between the two groups. While the non-concussed participants were more able to identify, focus, and fixate at the hazardous area, the concussed participants showed significantly more frequent distracted eye glances, were less able to focus, and showed less deliberate eye movement. The results support research results indicating the patients with concussion symptoms have great difficulty keeping their eyes focused on targets (Katrahmani \& Romoser, 2018b, 2018a). One of the potential reasons might be the effects that a concussion has on the brain.

Analyzing participants' data from the eye-tracker, we can conclude that it is difficult to interpret if a concussed patient is fit-to-drive. For instance, the level of impairment in hazard anticipation skills is not very clear. It is due to the fact that in the simulator scenarios, the hazards presented were not differentiated by the severity of danger. For instance, in the situation where a driver was able to identify a hazard and was not successful to recognize the next hazard, and another driver behaved the opposite, both were measured as one correct hazard anticipation and one missed hazard. However, the risk level and the consequences of each hazard has not been identified. In general, decision making regarding whether or not a person is fit-to-drive needs more research focusing on different aspects of driving skills.

In conclusion, further investigation on the effects of a concussion on the driving behavior of the teens is critically important. This experiment has demonstrated that using the eye-tracking data is a useful way to find the residual damage of a concussion on our study participants. The results of this study suggest a need for further research on teen drivers after receiving a concussion.

\section{REFERENCES}

Bazarian, J. J., Veazie, P., Mookerjee, S., \& Lerner, E. B. (2006). Accuracy of mild traumatic brain injury case ascertainment using ICD-9 codes. Academic Emergency Medicine, 13(1), $31-38$.

Bottari, C., Lamothe, M.-P., Gosselin, N., Gélinas, I., \& Ptito, A. (2012). Driving difficulties and adaptive strategies: the perception of individuals having sustained a mild traumatic brain injury. Rehabilitation Research and Practice, 2012.

Brouwer, Wiebo H., \& Withaar, F. K. (1997). Fitness to drive after traumatic brain injury. Neuropsychological Rehabilitation, 7(3), 177-193.

Chrisman, S. P., Quitiquit, C., \& Rivara, F. P. (2013). Qualitative study of barriers to concussive symptom reporting in high school athletics. Journal of Adolescent Health, 52(3), 330-335.

Gregory, D. R. (1981). The physician's role in highway safety-reporting requirements. American Association for Automotive Medicine Quarterly Jurnal, 3(4), 29-33.

Halstead, M. E., \& Walter, K. D. (2010). Sport-related concussion in children and adolescents. Pediatrics, 126(3), 597-615. 
Katrahmani, A., \& Romoser, M. (2018a). Diagnosing a Concussion by Testing Horizontal Saccades Using an Eye-Tracker. In International Conference on Applied Human Factors and Ergonomics (pp. 278-283). Springer.

Katrahmani, A., \& Romoser, M. (2018b). Implementing the Horizontal Vestibular Ocular Reflex Test While Using an Eye-Tracker as an Assessment Tool for Concussions Diagnosis. In International Conference on Applied Human Factors and Ergonomics (pp. 189-195). Springer.

Langlois, J. A., Rutland-Brown, W., \& Thomas, K. E. (2006). Traumatic brain injury in the United States; emergency department visits, hospitalizations, and deaths.

Novack, T. A., Baños, J. H., Alderson, A. L., Schneider, J. J., Weed, W., Blankenship, J., \& Salisbury, D. (2006). UFOV performance and driving ability following traumatic brain injury. Brain Injury, 20(5), 455-461.

Preece, M. H., Geffen, G. M., \& Horswill, M. S. (2013). Return-to-driving expectations following mild traumatic brain injury. Brain Injury, 27(1), 83-91.

Preece, M. H., Horswill, M. S., \& Geffen, G. M. (2010). Assessment of drivers' ability to anticipate traffic hazards after traumatic brain injury. Journal of Neurology, Neurosurgery \& Psychiatry, jnnp-2010.

Register-Mihalik, J. K., Guskiewicz, K. M., McLeod, T. C. V., Linnan, L. A., Mueller, F. O., \& Marshall, S. W. (2013). Knowledge, attitude, and concussion-reporting behaviors among high school athletes: a preliminary study. Journal of Athletic Training, 48(5), 645-653.

Rose, S. C., Weber, K. D., Collen, J. B., \& Heyer, G. L. (2015). The diagnosis and management of concussion in children and adolescents. Pediatric Neurology, 53(2), 108-118.

Schneider, J. J., \& Gouvier, W. D. (2005). Utility of the UFOV test with mild traumatic brain injury. Applied Neuropsychology, 12(3), 138-142.

Shenouda, C., Hendrickson, P., Davenport, K., Barber, J., \& Bell, K. R. (2012). The effects of concussion legislation one year later-what have we learned: a descriptive pilot survey of youth soccer player associates. $P M \& R, 4(6), 427-435$.

Sivak, M., Olson, P. L., Kewman, D. G., Won, H., \& Henson, D. L. (1981). Driving and perceptual/cognitive skills: behavioral consequences of brain damage. Archives of Physical Medicine and Rehabilitation, 62(10), 476-483.

Stokx, L. C., \& Gaillard, A. W. (1986). Task and driving performance of patients with a severe concussion of the brain. Journal of Clinical and Experimental Neuropsychology, 8(4), 421436.

Torres, D. M., Galetta, K. M., Phillips, H. W., Dziemianowicz, E. M. S., Wilson, J. A., Dorman, E. S., ... Balcer, L. J. (2013). Sports-related concussion Anonymous survey of a collegiate cohort. Neurology: Clinical Practice, 3(4), 279-287.

Webster, J. S., Rapport, L. J., Godlewski, M. C., \& Abadee, P. S. (1994). Effect of attentional bias to right space on wheelchair mobility. Journal of Clinical and Experimental Neuropsychology, 16(1), 129-137. 